\title{
On conformal Jordan cells of finite and infinite rank
}

\author{
Jørgen Rasmussen \\ Centre de recherches mathématiques, Université de Montréal \\ Case postale 6128, succursale centre-ville, Montréal, Qc, Canada H3C 3J7 \\ rasmusse@crm.umontreal.ca
}

\begin{abstract}
This work concerns in part the construction of conformal Jordan cells of infinite rank and their reductions to conformal Jordan cells of finite rank. It is also discussed how a procedure similar to Lie algebra contractions may reduce a conformal Jordan cell of finite rank to one of lower rank. A conformal Jordan cell of rank one corresponds to a primary field. This offers a picture in which any finite conformal Jordan cell of a given conformal weight may be obtained from a universal covering cell of the same weight but infinite rank.
\end{abstract}

MSC (2000): $81 \mathrm{~T} 40$

Keywords: Logarithmic conformal field theory, Jordan cells. 
Logarithmic conformal field theory is essentially based on the appearance of conformal Jordan cells, or Jordan cells for short, in the spectrum of fields. We refer to [1] for the first systematic study of logarithmic conformal field theory, and to [2, 3, 4] for recent reviews on the subject. The number of fields making up a Jordan cell is called the rank of the cell. Only Jordan cells of finite rank have been discussed in the literature. An objective of this work is to propose an extension to Jordan cells of infinite rank.

We shall also discuss how Jordan cells of finite or infinite rank may be reduced to Jordan cells of lower rank. In this regard, a Jordan cell of rank one merely corresponds to an ordinary primary field. The reductions of Jordan cells of finite rank are governed by a procedure resembling Lie algebra contractions. A related procedure has recently been employed in [5, 6, 7, 8, and in its various guises it constitutes a promising new approach to logarithmic conformal field theory.

A Jordan cell of rank $r=\rho+1$ consists of a primary field, $\Psi_{0}$, and $\rho$ logarithmic and non-primary partners, $\Psi_{1}, \ldots, \Psi_{\rho}$, satisfying 9

$$
T(z) \Psi_{j}(w)=\frac{\Delta \Psi_{j}(w)+\left(1-\delta_{j, 0}\right) \Psi_{j-1}(w)}{(z-w)^{2}}+\frac{\partial_{w} \Psi_{j}(w)}{z-w}, \quad j=0,1, \ldots, \rho
$$

where $\Psi_{-1} \equiv 0 . \quad T$ is the energy-momentum tensor whose modes, $L_{n}$, generate the Virasoro algebra

$$
\left[L_{n}, L_{m}\right]=(n-m) L_{n+m}+\frac{c}{12} n\left(n^{2}-1\right) \delta_{n+m, 0}
$$

with central charge $c$. In terms of these, (11) reads

$$
\left[L_{n}, \Psi_{j}(z)\right]=\left\{z^{n+1} \partial_{z}+\Delta(n+1) z^{n}\right\} \Psi_{j}(z)+(n+1) z^{n} \Psi_{j-1}(z)
$$

The two-point functions of the fields comprising the Jordan cell are

$$
\begin{aligned}
\left\langle\Psi_{i}(z) \Psi_{j}(w)\right\rangle & =0, \quad i+j<\rho \\
\left\langle\Psi_{i}(z) \Psi_{\rho}(w)\right\rangle & =\frac{\sum_{m=0}^{i} \frac{(-2)^{m}}{m !} A_{i-m}(\ln (z-w))^{m}}{(z-w)^{2 \Delta}} \\
\left\langle\Psi_{i}(z) \Psi_{j}(w)\right\rangle & =\left\langle\Psi_{i+j-\rho}(z) \Psi_{\rho}(w)\right\rangle \\
& =\frac{\sum_{m=0}^{i+j-\rho} \frac{(-2)^{m}}{m !} A_{i+j-\rho-m}(\ln (z-w))^{m}}{(z-w)^{2 \Delta}}, \quad i+j \geq \rho
\end{aligned}
$$

with structure constants $A_{j}, j=0, \ldots, \rho$. As already mentioned, it is seen that a rank-one Jordan cell is simply a primary field.

For later convenience, we now re-express (11) using the following self-explanatory matrix notation

$$
T(z) \Psi(w)=\frac{\Delta \Psi(w)+P \Psi(w)}{(z-w)^{2}}+\frac{\partial_{w} \Psi(w)}{z-w}
$$


where we have introduced the off-diagonal $r \times r$ matrix

$$
P=\left(\begin{array}{ccccccc}
0 & 0 & 0 & \ldots & 0 & 0 & 0 \\
1 & 0 & 0 & \ldots & 0 & 0 & 0 \\
0 & 1 & 0 & \ldots & 0 & 0 & 0 \\
\vdots & & & \vdots & & & \vdots \\
\vdots & & & \vdots & & & \vdots \\
0 & 0 & 0 & \ldots & 1 & 0 & 0 \\
0 & 0 & 0 & \ldots & 0 & 1 & 0
\end{array}\right)
$$

such that $P_{i, j}=\delta_{i, j+1}$. To emphasize the size of a matrix, we shall sometimes write $P_{r \times r}$ or $\Psi_{r \times 1}$, for example.

Our first objective is to discuss how a Jordan cell of rank $r=\rho+1$ may be reduced to a Jordan cell of rank $r^{\prime}<r$. If the difference $r-r^{\prime}=2 q$ is even, the reduction is straightforward as one may simply set

$$
\Psi_{0} \equiv \ldots \equiv \Psi_{q-1} \equiv \Psi_{\rho-q+1} \equiv \ldots \equiv \Psi_{\rho} \equiv 0
$$

The surviving fields, $\Psi_{q}, \ldots, \Psi_{\rho-q}$, are seen to form a Jordan cell of rank $r^{\prime}$ with $\Psi_{q}$ as its only primary member. It should be stressed that we in this work confine ourselves to considering Jordan cells from the point of view of conformal structure (11) and (chiral) two-point functions (4). It is immediately more complicated to describe the reduction if $r-r^{\prime}$ is odd. We shall focus on the case where this difference is one as all reductions may be constructed as concatenations of such unit reductions. This applies as well, of course, to the even case discussed above, but corresponds to an alternative scenario.

Let us consider the linear map

$$
\left(\begin{array}{c}
\Phi_{0 ; \epsilon} \\
\vdots \\
\Phi_{\rho ; \epsilon}
\end{array}\right)=\left(\begin{array}{ccc}
U_{0,0 ; \epsilon} & \ldots & U_{0, \rho ; \epsilon} \\
\vdots & & \vdots \\
U_{\rho, 0 ; \epsilon} & \ldots & U_{\rho, \rho ; \epsilon}
\end{array}\right)\left(\begin{array}{c}
\Psi_{0} \\
\vdots \\
\Psi_{\rho}
\end{array}\right)
$$

of the original representation of the Jordan cell given in terms of $\Psi$ to a new one in terms of $\Phi_{\epsilon}$. The map is governed by the matrix $U_{\epsilon}$ which is assumed invertible for non-vanishing $\epsilon$. We shall be interested in the limit ${ }^{1}$

$$
\Phi_{j}(z):=\lim _{\epsilon \rightarrow 0} \Phi_{j ; \epsilon}(z)
$$

and look for a map (8) that would result in a decoupling of the set $\Phi_{0}, \ldots, \Phi_{\rho}$ into a Jordan cell of rank $\rho$ consisting of the first $\rho$ fields, $\Phi_{0}, \ldots, \Phi_{\rho-1}$, and a primary field, $\Phi_{\rho}$, with vanishing two-point function.

\footnotetext{
${ }^{1}$ Note that $\Phi_{j}$ denotes a single field whereas $\Phi_{\epsilon}$ indicates a vector of fields denoted $\Phi_{j ; \epsilon}$. This is done to keep the notation simple, and will hopefully not cause confusion.
} 
To this end, we first consider

$$
\begin{aligned}
T(z) \Phi(w) & =\lim _{\epsilon \rightarrow 0}\left\{T(z) \Phi_{\epsilon}(w)\right\} \\
& =\frac{\Delta \Phi(w)+\lim _{\epsilon \rightarrow 0}\left\{U_{\epsilon} P U_{\epsilon}^{-1} \Phi_{\epsilon}(w)\right\}}{(z-w)^{2}}+\frac{\partial_{w} \Phi(w)}{z-w}
\end{aligned}
$$

from which it follows that we should require that

$$
\lim _{\epsilon \rightarrow 0}\left\{U_{r \times r ; \epsilon} P_{r \times r} U_{r \times r ; \epsilon}^{-1}\right\}=\left(\begin{array}{cc}
P_{\rho \times \rho} & 0_{\rho \times 1} \\
0_{1 \times \rho} & 0_{1 \times 1}
\end{array}\right)
$$

Likewise, from

$$
\left\langle\Phi_{i}(z) \Phi_{j}(w)\right\rangle=\lim _{\epsilon \rightarrow 0}\left\langle\Phi_{i ; \epsilon}(z) \Phi_{j ; \epsilon}(w)\right\rangle
$$

it follows that we should impose the conditions

$$
\begin{aligned}
& \lim _{\epsilon \rightarrow 0}\left\{\sum_{k, l=0}^{\rho} U_{i, k ; \epsilon} U_{j, l ; \epsilon} \sum_{m=0}^{k+l-\rho} \frac{(-2)^{m}}{m !} A_{k+l-\rho-m}(\ln (z-w))^{m}\right\} \\
& \quad= \begin{cases}\sum_{m=0}^{i+j-\rho+1} \frac{(-2)^{m}}{m !} A_{i+j-\rho+1-m}(\ln (z-w))^{m}, & 0 \leq i, j<\rho \\
0, & i=\rho \text { or } j=\rho\end{cases}
\end{aligned}
$$

We have found the following solution for the matrix $U_{r \times r ; \epsilon}$ :

$$
\begin{aligned}
U_{i, j ; \epsilon} & =0, \quad i+1<j \\
U_{i, i+1 ; \epsilon} & =\frac{\epsilon}{2} \\
U_{i, j ; \epsilon} & =\frac{(-1)^{i-j}}{i-j+1}\left(\begin{array}{c}
2(i-j) \\
i-j
\end{array}\right) \epsilon^{-2(i-j)-1}, \quad j \leq i<\rho \\
U_{\rho, j ; \epsilon} & =\delta_{j, 0}
\end{aligned}
$$

which for lower rank reads

$$
U_{2 \times 2 ; \epsilon}=\left(\begin{array}{cc}
1 / \epsilon & \epsilon / 2 \\
1 & 0
\end{array}\right), \quad U_{3 \times 3 ; \epsilon}=\left(\begin{array}{ccc}
1 / \epsilon & \epsilon / 2 & 0 \\
-1 / \epsilon^{3} & 1 / \epsilon & \epsilon / 2 \\
1 & 0 & 0
\end{array}\right)
$$

and

$$
U_{4 \times 4 ; \epsilon}=\left(\begin{array}{cccc}
1 / \epsilon & \epsilon / 2 & 0 & 0 \\
-1 / \epsilon^{3} & 1 / \epsilon & \epsilon / 2 & 0 \\
2 / \epsilon^{5} & -1 / \epsilon^{3} & 1 / \epsilon & \epsilon / 2 \\
1 & 0 & 0 & 0
\end{array}\right)
$$


Before proving that (14) indeed constitutes a solution, it is useful to note that

$$
U_{(r-1) \times(r-1) ; \epsilon}=R_{(r-1) \times r, r-1} U_{r \times r ; \epsilon} C_{r \times(r-1), r}
$$

and

$$
R_{(r-2) \times(r-1), r-1} U_{(r-1) \times(r-1) ; \epsilon}=R_{(r-2) \times(r-1), 1} R_{(r-1) \times r, r} U_{r \times r ; \epsilon} C_{r \times(r-1), 1}
$$

Here we have introduced the matrix

$$
R_{(n-1) \times n, i}=\left(\begin{array}{ccc}
I_{(i-1) \times(i-1)} & 0_{(i-1) \times 1} & 0_{(i-1) \times(n-i)} \\
0_{(n-1) \times(i-1)} & 0_{(n-i) \times 1} & I_{(n-i) \times(n-i)}
\end{array}\right)
$$

annihilating the $i$ th row in a matrix $M_{n \times m}$ by multiplication from the left. Likewise, annihilation of the $j$ th column is governed by multiplication from the right by the matrix

$$
C_{m \times(m-1), j}=\left(R_{(m-1) \times m, j}\right)^{t}=\left(\begin{array}{cc}
I_{(j-1) \times(j-1)} & 0_{(j-1) \times(m-j)} \\
0_{1 \times(j-1)} & 0_{1 \times(m-j)} \\
0_{(m-j) \times(j-1)} & I_{(m-j) \times(m-j)}
\end{array}\right)
$$

One does not have to worry about working out explicitly the inverse of $U_{\epsilon}$ and subsequently considering the limit in (11), since it turns out that

$$
U_{r \times r ; \epsilon} P_{r \times r}=\left(\begin{array}{cc}
P_{\rho \times \rho} & 0_{\rho \times 1}^{(\epsilon)} \\
0_{1 \times \rho} & 0_{1 \times 1}
\end{array}\right) U_{r \times r ; \epsilon}, \quad 0_{\rho \times 1}^{(\epsilon)}=\left(\begin{array}{c}
\epsilon / 2 \\
0_{(\rho-1) \times 1}
\end{array}\right)
$$

from which the condition (111) follows immediately. The refinement (21) of (11) follows itself from combining (17) and (18).

Now, the conditions (13) are easily verified for $i=\rho$ or $j=\rho$, so we may focus on $0 \leq i, j<\rho$. Using, among other things, that $k+l \geq \rho$ on the left-hand side of (13), we find that (13) is equivalent to demanding

$$
\lim _{\epsilon \rightarrow 0}\left\{\sum_{n=N-j-1}^{i+1} U_{i, n ; \epsilon} U_{j, N-n ; \epsilon}\right\}=\delta_{N, i+j+1}
$$

The non-trivial part of this reads

$$
\sum_{n=1}^{N} \beta_{N, n}=S_{N}
$$

where

$$
\beta_{N, n}=\frac{1}{n(N-n+1)}\left(\begin{array}{c}
2 N-2 n \\
N-n
\end{array}\right)\left(\begin{array}{c}
2 n-2 \\
n-1
\end{array}\right), \quad S_{N}=\frac{1}{N+1}\left(\begin{array}{c}
2 N \\
N
\end{array}\right)
$$


To prove (23) by induction in $N$, we first verify that it is satisfied for $N=1$. We then try to find $x_{2}, \ldots, x_{N}$ such that

$$
\begin{aligned}
\beta_{N+1,1}+x_{2} \beta_{N+1,2} & =\frac{S_{N+1}}{S_{N}} \beta_{N, 1} \\
\left(1-x_{n}\right) \beta_{N+1, n}+x_{n+1} \beta_{N+1, n+1} & =\frac{S_{N+1}}{S_{N}} \beta_{N, n}, \quad n=2, \ldots, N-1 \\
\left(1-x_{N}\right) \beta_{N+1, N}+\beta_{N+1, N+1} & =\frac{S_{N+1}}{S_{N}} \beta_{N, N}
\end{aligned}
$$

since this would imply that

$$
\sum_{n=1}^{N+1} \beta_{N+1, n}=\frac{S_{N+1}}{S_{N}} \sum_{n=1}^{N} \beta_{N, n}=S_{N+1}
$$

as required to satisfy the induction step. The last equality follows from (23) which now serves as the induction assumption. The existence of a solution to the set of linear equations (25) is non-trivial since there is one more constraint than free parameters. It is not hard to verify, though, that the set

$$
x_{n}=\frac{n(n-1)(3 N-2 n+4)}{N(N+1)(N+2)}, \quad n=2, \ldots, N
$$

solves (25) and hence the induction step.

This completes the proof that the map (8) with $U_{\epsilon}$ given by (14) reduces the Jordan cell $\left(\Psi_{0}, \ldots, \Psi_{\rho}\right)$ to the Jordan cell $\left(\Phi_{0}, \ldots, \Phi_{\rho-1}\right)$, in the limit $\epsilon \rightarrow 0$. By construction, the structure constants of the resulting lower-rank Jordan cell are thereby inherited from the original higher-rank Jordan cell. This should not be regarded as a limitation of the procedure since the constants may be modified by a further re-scaling of the new fields.

The conventional representation of a Jordan cell as given above in (11) and (4) does not seem to allow a naive extension to infinite rank. This may explain why Jordan cells of infinite rank so far have managed to elude explicit construction. To remedy this, we here suggest to first re-label the Jordan cell subtracting $\rho / 2$ from the index of the fields:

$$
r=\rho+1 \text { even : } \quad \Psi_{-\rho / 2}, \Psi_{-\rho / 2+1}, \ldots, \Psi_{-1 / 2}, \Psi_{1 / 2}, \ldots, \Psi_{\rho / 2}
$$

and

$$
r=\rho+1 \text { odd }: \quad \Psi_{-\rho / 2}, \Psi_{-\rho / 2+1}, \ldots, \Psi_{-1}, \Psi_{0}, \Psi_{1}, \ldots, \Psi_{\rho / 2}
$$

In either case, the field $\Psi_{-\rho / 2}$ is the only primary one in the full Jordan cell. The strong resemblance of (28) and (29) to $s u(2)$ representations of spin $\rho / 2$ will be discussed elsewhere.

The limit where the rank approaches infinity is more natural in the new setting given by (28) and (29). Namely, let us consider the two infinite sets of fields $\left\{\Psi_{j}\right\}$ where either 
$j \in(\mathbb{Z}+1 / 2)$ or $j \in \mathbb{Z}$, corresponding to extensions of the two possibilities (28) and (29), respectively. In either case, we have

$$
T(z) \Psi_{j}(w)=\frac{\Delta \Psi_{j}(w)+\Psi_{j-1}(w)}{(z-w)^{2}}+\frac{\partial_{w} \Psi_{j}(w)}{z-w}
$$

whereas the two-point functions shall be defined by

$$
\begin{aligned}
& \left\langle\Psi_{i}(z) \Psi_{j}(w)\right\rangle=0, \quad i+j<0 \\
& \left\langle\Psi_{i}(z) \Psi_{j}(w)\right\rangle=\frac{\sum_{m=0}^{i+j} \frac{(-2)^{m}}{m !} A_{i+j-m}(\ln (z-w))^{m}}{(z-w)^{2 \Delta}}, \quad i+j \geq 0
\end{aligned}
$$

Indeed, it may be verified straightforwardly that these two-point functions are quasiconformally or projectively invariant in the sense that insertions of $L_{0, \pm 1}$ vanish. It is noted that

$$
\left\langle\Psi_{i+k}(z) \Psi_{j-k}(w)\right\rangle=\left\langle\Psi_{i}(z) \Psi_{j}(w)\right\rangle
$$

for all integer $k$, and that, unlike the finite case, there is no primary field in such a Jordan cell of infinite rank.

From (31) it seems natural to define the conjugate field to $\Psi_{j}$ as $\Psi_{-j}$. This would ensure that two-point functions of conjugate fields would be given by the familiar pole structure only:

$$
\left\langle\Psi_{j}(z) \Psi_{-j}(w)\right\rangle=\frac{A_{0}}{(z-w)^{2 \Delta}}
$$

A sensible construction would thus presuppose $A_{0} \neq 0$. With this definition of conjugate fields, we see that the two types of infinite Jordan cells may be distinguished by whether or not they contain a self-conjugate field, corresponding to $j \in \mathbb{Z}$ or $j \in(\mathbb{Z}+1 / 2)$, respectively. Due to the similarity with (29) and (28), we shall call the two types odd or even, respectively. It is stressed, though, that for a given finite rank there is only one Jordan cell. It may be represented by $\left(\Psi_{0}, \ldots, \Psi_{r-1}\right)$ as in (11) and (41), or by (28) or (29) depending on (the parity of) the rank.

An infinite Jordan cell as defined above reduces to an ordinary Jordan cell of finite rank, $r=\rho+1$, if one introduces the parameter $\rho$ and defines

$$
\Psi_{j} \equiv 0, \quad|j| \geq \rho / 2+1
$$

This requires that the original Jordan cell of infinite rank is of the same type as the parity of the rank of the resulting finite cell. From the reduction procedure above, on the other hand, we know how to reduce a Jordan cell of finite rank to one of any lower rank. This seems to suggest that there are two possible parent Jordan cells of infinite rank from which any finite Jordan cell can be obtained, provided, of course, they are of the same conformal weight. From this perspective, one could choose either of the two types or proposals and consider it the 'universal covering cell' of a given conformal weight. A reason for favouring one type over the other does not present itself immediately. It 
is not even clear that any of them should be excluded. The existence or non-existence of a self-conjugate field, which constitutes the characterizing difference between the two types, may play an important role in some contexts.

In summary, we have found that Jordan cells of a given common conformal weight are naturally organized in an infinite hierarchy labeled by their rank. A Jordan cell of rank $r$ thus appears at level $r$ in the hierarchy. The Jordan cells are nested in the sense that a Jordan cell of rank $r^{\prime}$ may be obtained by reduction of a Jordan cell of rank $r$ provided $r^{\prime}<r$. Two types of Jordan cells of infinite rank have been proposed as the universal covering cell from which any finite Jordan cell may be obtained by reduction.

Finally, our construction of Jordan cells of infinite rank and their reductions may be extended to $N=1$ superconformal field theory. This also applies to the reduction procedure of Jordan cells of finite rank, and will be addressed elsewhere.

\section{Acknowledgements}

The author thanks Michael Flohr for comments.

\section{References}

[1] Gurarie, V.: Logarithmic operators in conformal field theory, Nucl. Phys. B 410 (1993), 535-549.

[2] Flohr, M.: Bits and pieces in logarithmic conformal field theory, Int. J. Mod. Phys. A 18 (2003), 4497-4592.

[3] Gaberdiel, M.R.: An algebraic approach to logarithmic conformal field theory, Int. J. Mod. Phys. A 18 (2003), 4593-4638.

[4] Nichols, A.: $S U(2)_{k}$ logarithmic conformal field theory, Ph.D. thesis (University of Oxford, 2002), hep-th/0210070

[5] Bakas, I., Sfetsos, K.: PP-waves and logarithmic conformal field theories, Nucl. Phys. B 639 (2002), 223-240.

[6] Rasmussen, J.: On string backgrounds and (logarithmic) CFT, based on contribution to IX-th Workshop on Mathematical Physics, Rabat, February 2004, African Jour. Math. Phys. 1 (2004), 171-175, hep-th/0404226.

[7] Rasmussen, J.: Logarithmic limits of minimal models, Nucl. Phys. B 701 (2004), 516-528.

[8] Rasmussen, J.: Jordan cells in logarithmic limits of conformal field theory, preprint, hep-th/0406110. 
[9] Rahimi Tabar, M.R., Aghamohammadi, A., Khorrami, M.: The logarithmic conformal field theories, Nucl. Phys. B 497 (1997), 555-566. 